\title{
Alcoolismo após cirurgia bariátrica: relato de caso
}

\author{
Alcoholism following bariatric surgery: case report \\ Juliana Garbayo dos Santos', Marcelo Santos Cruz'
}

\section{RESUMO}

Objetivo: Discutir, a partir do relato de um caso de alcoolismo iniciado após tratamento cirúrgico de obesidade mórbida, a hipótese da "transferência de compulsão". Segundo essa teoria, obesos submetidos à cirurgia bariátrica tendem a transferir sua "compulsão" dos alimentos para o álcool ou outras drogas, aumentando o risco de desenvolvimento de transtornos relacionados a substâncias. Evidências de que a alimentação hedônica (caracterizada pelo consumo exagerado de alimentos palatáveis na ausência de fome) é regulada pelos mesmos sistemas cerebrais envolvidos no alcoolismo, aliadas a relatos anedóticos de alcoolismo após cirurgia bariátrica, alimentam essa hipótese. Métodos: Relato de caso individual. O paciente concordou com a publicação do relato de caso e assinou um Termo de Consentimento Livre e Esclarecido. Resultados: Homem de 25 anos, sem histórico prévio de abuso de álcool, evoluiu com alcoolismo grave quatro anos após a cirurgia bariátrica. Havia antecedentes familiares de transtornos relacionados a substâncias. Conclusão: A cirurgia bariátrica (particularmente com derivação em Y de Roux) pode alterar o metabolismo do etanol e implicar mudanças no estilo de vida e no nível de estresse. Somados, esses

\section{Palavras-chave}

Obesidade, obesidade mórbida, cirurgia bariátrica, alcoolismo, transtornos induzidos por álcool. fatores podem contribuir para o surgimento de padrões nocivos de consumo de álcool; entretanto, é incontestável que a correção da obesidade traz enormes benefícios para as diversas áreas da vida do paciente. A detecção de fatores de risco para abuso e dependência alcoólica, como história familiar positiva, é essencial para identificar pacientes que necessitarão maior aconselhamento pré-operatório e seguimento pós-operatório cuidadoso.

\section{ABSTRACT}

Objective: To discuss, based on the report of a case of alcoholism that started after surgical treatment of morbid obesity, the idea of the "transfer of compulsion". According to this theory, obese people subjected to bariatric surgery tend to transfer their "compulsion" from food to alcohol or other drugs, increasing the risk of developing substance-related disorders. Evidence that hedonic feeding behavior (described as overeating of palatable foods in the absence of hunger) is regulated by the same brain systems involved in alcoholism, allied to anecdotal reports of alcoholism after bariatric surgery, serve to support this hypothesis. Methods: Single case report. Patient agreed with the publication of the case report and signed the Informed Consent Form. Results: 25 year-old male, with no history of alcohol abuse, developed heavy drinking habits four years after bariatric surgery.

1 Universidade Federal do Rio de Janeiro (UFRJ), Instituto de Psiquiatria (IPUB).
Recebido em

Aprovado em

10/11/2016
Endereço para correspondência: Juliana Garbayo dos Santos Rua Visconde de Pirajá, 142/902, Ipanema - 22410-000 - Rio de Janeiro, RJ, Brasil. E-mail:dra.juliana.garbayo@gmail.com 


\section{Keywords}

Obesity, obesity, morbid,

bariatric surgery,

alcoholism, alcohol-

induced disorders.
There was family history of substance-related disorders. Conclusion: Bariatric surgery (particularly Roux-en-Y gastric bypass) may alter ethanol metabolism and implicate changes in life style and stress levels. Put together, these factors can contribute to the development of harmful alcohol consumption patterns that need to be considered in contrast with the great benefits that surgical correction of obesity brings to several areas of patient's life. Detecting risk factors for alcohol abuse and dependency, such as positive family history, is essential to identify patients who will need greater pre-operative counselling and careful post-operative follow-up.

\section{INTRODUÇÃO}

O comportamento alimentar hedônico envolve busca e consumo de alimentos palatáveis mesmo na ausência de fome, está associado à obesidade e é regulado pelos mesmos sistemas cerebrais implicados no alcoolismo'. Relataremos o caso de um paciente que desenvolveu grave dependência alcoólica após cirurgia bariátrica (CB), discutindo a crença do senso comum de que obesos mórbidos "transferem" a compulsão para outras substâncias quando impedidos de comer excessivamente.

\section{RELATO DO CASO}

J., masculino, 25 anos, autônomo (trabalha em casa, pela internet). Aos 13 anos, foi violentado sexualmente por desconhecidos. Recebeu apoio psicológico e familiar, mas passou a apresentar recusa escolar, concluindo seus estudos a distância. Nos seis anos seguintes, engordou 60 kg (até então, tinha peso normal para altura e idade). Além de seis refeições diárias, comia biscoitos, sanduíches e nuggets fritos inúmeras vezes por dia; esse consumo ocorria ora em quantidade moderada, junto com familiares, ora em quantidade exagerada, escondido e com sensação de descontrole. Pelo significativo ganho ponderal e pelo histórico do abuso, a família procurou ajuda psiquiátrica para J. e este fez uso, dos 16 aos 18 anos, de baixas doses de sertralina ( $25 \mathrm{mg} / \mathrm{dia})$, topiramato (máximo 25 mg/dia) e zolpidem. Aos 19 anos, atingiu $140 \mathrm{~kg}$ (altura: 1,73 m, índice de massa corporal (IMC): $46 \mathrm{~kg} / \mathrm{m}^{2}$ ) e submeteu-se à gastroplastia com derivação em $Y$ de Roux. Perdeu $30 \mathrm{~kg}$ nos dois anos seguintes e continuou emagrecendo até atingir, aos 22 anos, $I M C=26,7 \mathrm{~kg} / \mathrm{m}^{2}$. Aos 23 anos, foi para Irlanda estudar e trabalhar. Tão logo se mudou, começou a beber vodka, duas a três vezes por semana (aproximadamente 7 doses por dia). Nos sete meses seguintes, abandonou o emprego e o curso e passou a beber diariamente. Começou a apresentar tremores e náuseas. Prosseguiu nesse padrão por mais dois meses, até acordar "vendo aranhas" e "tremendo demais". Levado à emergência, foi indicada internação por delirium tremens. Voltou ao Brasil e, poucos meses depois, buscou atendimento psiquiátrico referindo "medo de morrer de tanto beber". Bebia $500 \mathrm{ml}$ de vodka por dia. Quando os tremores agravavam a ponto de dificultar atividade laboral (digitação), mantinha-se abstinente por até uma semana. Bebia entre 1 e 4 horas da manhã, no quarto; dormia até 13 horas e acordava com mal-estar, náuseas, fraqueza e "palpitações". Em uma queda alcoolizado "bateu a cabeça no chão" (sem perda da consciência); em outra, sofreu corte profundo no antebraço. Internação hospitalar foi indicada, mas recusada. Foram prescritos escitalopram, zolpidem e tiamina. Recusou topiramato, alegando efeito colateral quando do uso prévio. Naltrexona foi descartada após exames revelando aumento de aminotransferases. Demais enzimas hepáticas, hemograma, função renal e tireoidiana, lipidograma e glicose normais. Após o esclarecimento quanto ao dano hepático causado pelo seu consumo de álcool, aceitou internação especializada. História familiar: mãe submetida à CB; pai obeso, com depressão; avô paterno (falecido) e três primas com transtornos por álcool e drogas.

\section{DISCUSSÃO}

A obesidade (IMC $\geq 30 \mathrm{~kg} / \mathrm{m}^{2}$ ) afeta $15,8 \%$ dos adultos brasileiro ${ }^{2}$ e vem se tornando cada vez mais prevalente ${ }^{2}$. O tratamento cirúrgico é altamente eficaz ${ }^{3}$ e indicado na obesidade grave sem resposta a tratamento conservador prévio ou com doenças desencadeadas/agravadas pela obesidade.

O álcool é a substância psicoativa (SPA) mais consumida no Brasil e no mundo, mas seu uso indevido é fator de risco para doenças, acidentes, violência, absenteísmo, invalidez e morte prematuras ${ }^{4}$. A dependência se caracteriza por um padrão no qual beber é priorizado apesar das consequências adversas e em detrimento de atividades antes valorizadas. Afeta 12,3\% da população brasileira acima de 12 anos, particularmente os homens $(19,5 \%)^{4}$.

Alimentos altamente palatáveis agem como drogas de abuso nos centros de prazer e recompensa cerebrais, gerando modificações praticamente indistinguíveis daquelas induzidas pelas substâncias psicoativas (SPA)'. O potencial de provocar dependência de uma substância depende de sua capacidade de agir sobre a neurotransmissão dopaminérgica na via mesocorticolímbica ${ }^{5}$, condição preenchida pelos doces, cujo consumo compulsivo estimula a liberação de dopamina (DA) no núcleo accumbens (NAC)'. Tanto as 
drogas quanto os alimentos altamente palatáveis tornam as vias do sistema de recompensa cerebral hiper-responsivas à substância de escolha (reforçando o uso) e hiporresponsivas a outros estímulos (reduzindo a capacidade de obter prazer de outras fontes) ${ }^{1,5}$. Decréscimos na função do córtex pré-frontal e orbitofrontal (comprometendo autocontrole) e redução persistente nos níveis de receptores D2, além de hiperatividade dos sistemas cerebrais de estresse, são alterações frequentemente encontradas entre dependentes de SPA ${ }^{5}$ que reforçam o uso da substância potencializando seus efeitos prazerosos e evitando o desprazer gerado pela sua privação (como a irritabilidade e desmotivação da abstinência). Não faltam exemplos de como isso ocorre na alimentação. Mulheres que desenvolvem marcada preferência por carboidratos experimentam fissura e, com a continuidade do uso, diminuição da capacidade desse alimento de aliviar a disforia' (tolerância). Ratos consumindo doces repetidamente também desenvolvem tolerância a seus efeitos ${ }^{1}$. Obesos têm expressão diminuída de receptores D2 no núcleo estriado e aumento do metabolismo do córtex somatossensorial em resposta a propriedades sensoriais da comida (alterações cerebrais que reforçam o comer e prejudicam a habilidade de obter prazer com outras atividades) ${ }^{1}$. Ratos obesos têm hipoatividade dopaminérgica cerebral e a comida aumenta a liberação de DA - o que não acontece com ratos não obesos, nos quais os alimentos não exibem essa propriedade ${ }^{1}$. 0 álcool e os alimentos gordurosos reforçam o consumo um do outro, estimulando a produção hipotalâmica de peptídeos orexígenos e mediando elevações na taxa de triglicerídeos circulantes ${ }^{1}$ que estimulam a ingesta calórica. Ratos obesos desenvolvem síndrome de abstinência opioide-like em resposta ao antagonista opioide naloxona'. Rituais de despedida (libações alcoólicas precedendo abstinência e "banquetes" antecipando dietas rígidas), episódios de consumo compulsivo (binge drinking e binge eating) e suscetibilidade ao estresse (condição que leva a maioria das pessoas a comer mais e a preferir alimentos hipercalóricos) são outros aspectos comuns entre obesidade e alcoolismo. Entretanto, poucas evidências objetivas sustentam a crença de que obesos submetidos à CB transferem a compulsão para outra substância ${ }^{6-9}$ e mesmo o aumento da prevalência de transtornos pelo uso de álcool nessa população é controverso. Alguns estudos apontaram prevalência de abuso de álcool entre candidatos à CB similar à da população geral ${ }^{8-11}$ e não observaram aumento significativo após o procedimento ${ }^{8-10}$, mas outros ${ }^{7}$ encontraram acréscimos que, embora pequenos (de 7,6\% para 9,6\% dois anos após a $C B$ ), podem significar muito em custo pessoal e social, considerando o aumento na quantidade de cirurgias realizadas. Reslan et al. encontraram prevalência de abuso de substâncias pós-CB de $14 \%$, dos quais $70 \%$ representavam casos novos ${ }^{12}$, isto é, indivíduos sem abuso prévio à cirurgia. Em dois terços desses casos, o quadro teve início após os 40 anos, fato incomum em um transtorno que inicia tipicamente na juventude.

Alguns estudos 6,7 detectaram aumento da prevalência de problemas relacionados ao álcool dois anos após a CB, particularmente a derivação em $Y$ de Roux ${ }^{6-8}$. Imediatamente após a CB, entretanto, a frequência do uso de álcool tende a diminuir ${ }^{6,10}$, provavelmente refletindo o conjunto de cuidados e orientações do pós-operatório imediato. Uma pesquisa ${ }^{9}$ não encontrou mudança no padrão de consumo após a CB, mas os pacientes referiram intoxicação com menores doses (maior sensibilidade) e significativamente mais dificuldade em controlar o uso (mudanças que podem ser secundárias à alteração no metabolismo do álcool após a (B).

Embora a ideia de "transferência de compulsão" sugira que a CB apenas restrinja fisicamente a ingesta alimentar, sem produzir mudanças sobre o comportamento compulsivo, estudos de neuroimagem ${ }^{13}$ sugerem que a CB (particularmente a derivação em $Y$ de Roux) reestrutura os sistemas neurais gerando uma profunda mudança de atitude em relação à comida.

Mesmo nos casos em que obesidade e alcoolismo parecem associados, é preciso cautela ao inferir as razões dessa associação. A possível existência de um fator de risco subjacente às duas condições (como abuso sexual) é uma hipótese: pelo menos um estudo ${ }^{14}$ encontrou significativamente mais história de alcoolismo (11,9\%) em obesos com relato de abuso do que naqueles sem esse histórico (0,8\%).

Apenas $0,7 \%$ a $1,7 \%{ }^{7,12,15}$ dos pacientes na fila para CB reportam problemas com álcool na época da avaliação pré-operatória, embora já tenha sido descrita prevalência ao longo da vida de 35,3\% entre pacientes submetidos à $\mathrm{CB}^{8}$. Essa disparidade pode refletir receio de ser excluído do procedimento e apontar para a necessidade de aprimorar a forma de avaliação.

Devemos considerar ainda que a CB altera o metabolismo do álcool, o que pode facilitar tolerância e demais fenômenos envolvidos na dependência ${ }^{9,15}$ e modifica fortemente o estilo de vida (inclusive com maior integração social), gerando um estresse agudo para o qual o paciente pode estar despreparado. Nosso paciente foi exposto a três fortes eventos estressores (abuso, emigração e cirurgia bariátrica) que, somados à história familiar positiva, podem tê-lo colocado sob risco aumentado de dependência do álcool.

\section{CONCLUSÃO}

O caso descrito exemplifica a necessidade de identificar pacientes com fatores de risco para abuso e dependência alcoólica após CB, como história familiar positiva, os quais necessitarão de assistências pré- e pós-operatória cuidadosas. Alterações do metabolismo do etanol, no estilo de vida e no nível de estresse são riscos que devem ser considerados diante dos enormes benefícios da correção da obesidade. 


\section{CONTRIBUIÇÕES INDIVIDUAIS}

Juliana Garbayo dos Santos - Foi responsável pelo acompanhamento e tratamento médico do paciente.

Marcelo Santos Cruz e Juliana Garbayo dos Santos - Analisaram e revisaram os dados clínicos, elaboraram o artigo e aprovaram a versão final submetida para publicação.

\section{CONFLITOS DE INTERESSE}

Os autores não têm conflitos de interesse a declarar.

\section{REFERÊNCIAS}

1. Blumenthal DM, Gold MS. Neurobiology of food addiction. Curr Opin Clin Nutr Metab Care. 2010;13:359-65.

2. Brasil. Ministério da Saúde: Secretaria de Vigilância em Saúde. Vigitel Brasil 2011: Vigilância de Fatores de Risco e Proteção para Doenças Crônicas por Inquérito Telefônico. Ministério da Saúde. 2012. p. 132.

3. Buchwald H, Avidor Y, Braunwald E, Jensen MD, Pories W, Fahrbach K, et al. Bariatric surgery: a systematic review and meta-analysis. JAMA. 2004;292:1724-37.

4. Brasil. Secretaria Nacional de Políticas sobre Drogas, IME-USP, Duarte PCAV, Stempliuk VA, Barroso LP. Relatório brasileiro sobre drogas. Ministério da Saúde. Brasília, 2009. 364 p. Disponível em: <www.senad.gov.br>. Acessado em: 10 nov. 2014.
5. Kalivas PW, Volkow ND. The neural basis of addiction: a pathology of motivation and choice. Am J Psychiatry. 2005;162:1403-13.

6. Conason A, Teixeira J, Hsu CH, Puma L, Knafo D, Geliebter A. Substance use following bariatric weight loss surgery. JAMA Surg. 2013;148:145-50.

7. King WC, Chen JW, Mitchell JE, Kalarchian MA, Steffen KJ, Engel SG, et al. Prevalence of alcohol use disorders before and after bariatric surgery. JAMA. 2012;307:2516-25.

8. Suzuki J, Haimovici F, Chang G. Alcohol use disorders after bariatric surgery. Obes Surg. 2012;22:201-7.

9. Ertelt TW, Mitchell JE, Lancaster K, Crosby RD, Steffen KJ, Marino JM. Alcohol abuse and dependence before and after bariatric surgery: a review of the literature and report of a new data set. Surg Obes Relat Dis. 2008:4:647-50.

10. Wee CC, Mukamal KJ, Huskey KW, Davis RB, Colten ME, Bolcic-Jankovic D, et al. High-risk alcohol use after weight loss surgery. Surg Obes Relat Dis. 2014;10:508-15.

11. Saules KK, Wiedemann A, Ivezaj V, Hopper JA, Foster-Hartsfield J, Schwarz D. Bariatric surgery history among substance abuse treatment patients: prevalence and associated features. Surg Obes Relat Dis. 2010;6:615-21.

12. Reslan S, Saules KK, Greenwald MK, Schuh LM. Substance misuse following Roux-en-Y gastric bypass surgery. Subst Use Misuse. 2014;49:405-17.

13. Tonelli H, Sartori FM, Marchesini JCD, Marchesini JB, Tonelli DG. Effects of bariatric surgery on the central nervous system and eating behavior in humans: a systematic review on the neuroimaging studies. J Bras Psiquiatr. 2013;62:297-305.

14. Gabert D, Majumdar RS, Sharma AM, Rueda-Clausen CF, Klarenbach SW, Birch DW, et al. Prevalence and predictors of self-reported sexual abuse in severely obese patients in a population-based bariatric program. J Obes. 2013(2013):374050.

15. Heinberg LJ, Ashton K, Coughlin J. Alcohol and bariatric surgery: review and suggested recommendations for assessment and management. Surg Obes Relat Dis. 2012;8:357-63. 